\title{
The Supernova/GRB Connection
}

\author{
P. Höflich ${ }^{1}$, D. Baade ${ }^{2}$, A. Khokhlov ${ }^{3}$, L. Wang ${ }^{4}$, and J.C. Wheeler ${ }^{1}$ \\ 1 Dept. of Astronomy, University of Texas, Austin, TX 78681, USA; \\ pah@astro.as.utexas.edu \& wheel@hej3.as.utexas. edu \\ 2 ESO, Karl-Schwarzschild-Str. 2, 85748 Garching, Germany; \\ pdbaadeQeso.org \\ 3 Naval Research Lab, Washington DC, USA; \\ ajk@lcp.nrl.navy.mil \\ 4 Lawrence Berkeley Lab, CA 94720, USA; \\ lifan@panisse.lbl.gov
}

Summary. We discuss the possible connection between supernova explosions (SN) and gamma-ray bursters (GRB) from the perspective of our current understanding of SN physics. Core collapse supernovae (SN) are the final stages of stellar evolution in massive stars during which the central region collapses, forms a neutron star (NS) or black hole, and the outer layers are ejected. Recent explosion scenarios assumed that the ejection is due to energy deposition by neutrinos into the envelope but detailed models do not produce powerful explosions. There is new and mounting evidence for an asphericity and, in particular, for axial symmetry in several supernovae which may be hard to reconcile within the spherical picture. The 3-D signatures are a key to understand core collapse supernovae and the GRB/SN connection. In this paper we study the effects and observational consequences of asymmetric explosions.

\section{Introduction}

Recently, the connection between supernovae (SN) and gamma-ray bursters (GRB) has been established with the observations of GRB 030329 and the identification of the underlying SN2003dh [25]. Further evidence is found in observations of the circumstellar surrounding in GRB 021004, the coincidence between SN1998bw and GRB 980425 [24], possible identification of the Fe K line in the X-rays, and some evidence that GRBs are related to star forming regions. There is a general agreement that the explosion of a massive star is caused by the collapse of its central parts into a neutron star or, for massive progenitors, into a black hole. The mechanism of the energy deposition into the envelope is still debated. For an overview, see [11]. In particular, the direct collapse of the central region a very massive star to a black hole and the connection with the newly found hypernovae is a very attractive option [34].

In recent years, there has been a mounting evidence that the explosions of massive stars (core collapse supernovae) are highly aspherical.

1. The spectra of core-collapse supernovae (e.g., SN1987A, SN1993J, SN1994I, SN1999em, SN2002ap) are significantly polarized at a level of 0.5 to $3 \%$ 

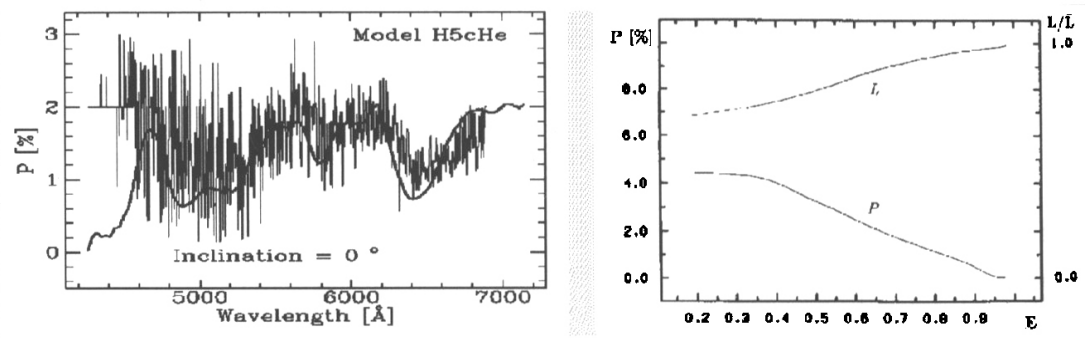

Fig. 1. Polarization spectrum for SN1993J for an axis ratio of $1 / 2$ for an oblate ellipsoid in comparison with observations by Trammell et al. [29] (left plot). On the right, the dependence of the continuum polarization (right) and directional dependence of the luminosity is shown as a function axis ratios for oblate ellipsoids seen from the equator (from $[8,10]$ ).

(e.g., Fig. 1 and $[4,8,14,22,30]$ ) indicating aspherical envelopes by factors of up to 2 (Fig. 2). The degree of polarization tends to vary inversely with the mass of the hydrogen envelope, being maximum for Type Ib/c events with no hydrogen [31]. For SNeII, Leonard et al. [19] and Wang et al. [32] showed that the polarization and, thus, the asphericity increase with time. Both trends suggest a connection of the asymmetries with the central engine. For supernovae with a good time and wavelength coverage, the orientation of the polarization vector tends to stay constant both in time and with wavelength. This implies that there is a global symmetry axis in the ejecta.

2. Observations of SN 1987A showed that radioactive material was brought to the hydrogen rich layers of the ejecta very quickly during the explosion (e.g., [19]).

3. The remnant of the Cas A supernova shows rapidly moving oxygen-rich matter outside the nominal boundary of the remnant and evidence for two oppositely directed jets of high-velocity material [5].

4. Recent X-ray observations with the CHANDRA satellite have shown an unusual distribution of iron and silicon group elements with large scale asymmetries in Cas A [13].

5. After the explosion, neutron stars are observed with high velocities, up to $1000 \mathrm{~km} / \mathrm{s}[27]$.

6. Direct HST-images from 11 June 2000, are able to resolve the inner debris of SN1987A showing its prolate geometry with an axis ratio of $\sim 2[11$, $32]$.

\section{Jet-Induced Supernovae and Observations}

In SNe, electron scattering is the main mechanism to polarize the light. It can be caused by asymmetries in the density, abundances or excitation structure 


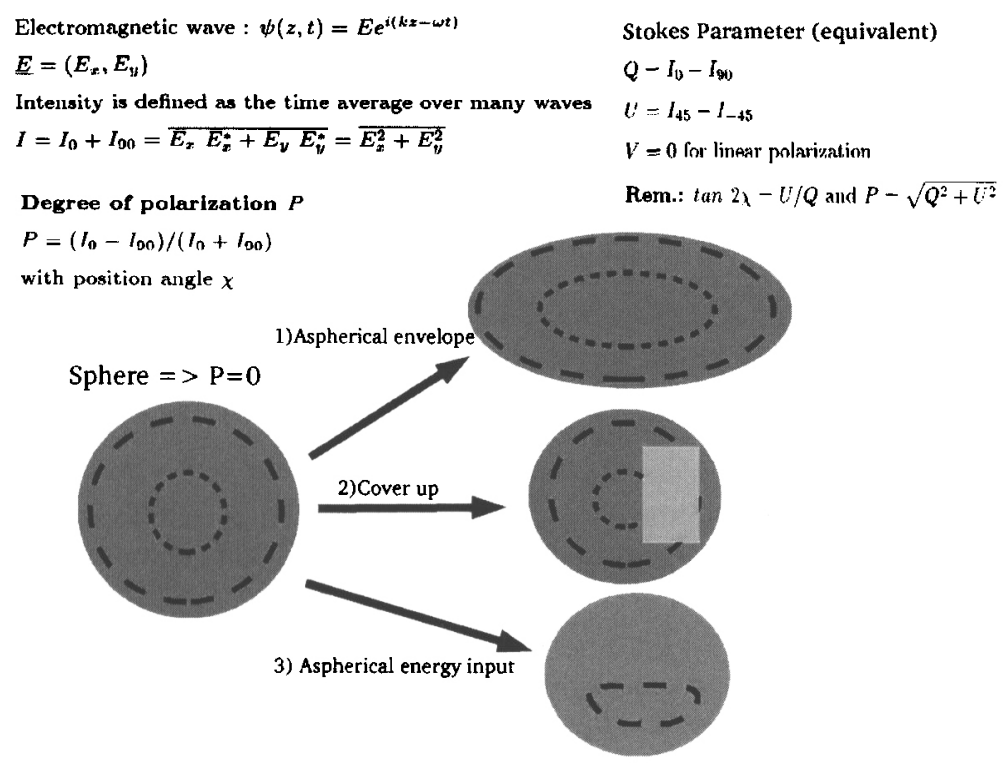

Fig. 2. Definition of the polarization and schematic diagram for its production. The dotted lines give the main orientation of the electrical vectors. For an unresolved sphere, the components cancel out. Three main mechanisms can be distinguished. $\bar{P}$ can be caused by 1) an aspherical envelope, 2) shading parts of the disk, or by 3) an aspherical excitation/ionization (from [9]).

of an envelope. In general, the supernovae ejecta cannot be spatially resolved. Although the light from different parts of a spherical disk is polarized, the resulting polarization $\bar{P}$ is zero for the integrated light (Fig. 2). To produce $\bar{P}$, three basic configurations must be considered. The envelope is aspherical, parts of the disk are shaded, and the envelope may be illuminated by an "offcenter" source. In case 2 , the shading may be either by a broad-band absorber such as dust or a specific line opacity. In the latter case, this would produce a change of $\bar{P}$ in a narrow line range (Fig. 4). In reality, a combination of all cases is realized (see below). Quantitative analyses are need to take into account that the continua and lines are formed in the same layers (e.g., Fig. 1).

We have numerically studied the explosion of core collapse supernovae caused by supersonic jets generated in the center of the star to ask for the jet-properties needed to reproduce observations. The initial stellar structures are based on stellar evolution calculations [26]. The explosion and jet propagation are calculated by a full 3-D code within a cubic domain. The Euler equations are integrated using an explicit, second-order accurate, Godunov type (PPM, [3]), adaptive-mesh-refinement, massively parallel, Fully- 
Threaded Tree (FTT) program, ALLA [16]. The subsequent evolution, LCs and spectra are calculated by using modules of our hydrodynamical radiation transport code for spherical and full 3-D (HYDRA) [12]. This code includes modules for hydrodynamics using PPM (without mesh refinement), detailed networks for nuclear processes and for atomic, non-LTE level populations, and radiation transport (with mesh-refinement). Its components have been used to carry out many of the previous calculations to analyze light curves, flux and polarization spectra of thermonuclear and supernovae ([7] ... [12]).

\subsection{General Results}

We simulated the process of the jet propagation through the star, the redistribution of elements, and radiation transport effects. Qualitatively, the jetinduced picture allows to reproduce the polarization observed in core collapse supernovae. Both asymmetric ionization and density/chemical distributions are crucial for the production of $P$. Even within the picture of jet-induced explosion, the latter effect alone cannot (!) account for the large $P$ produced in the intermediate H-rich layers of core-collapse $\mathrm{SN}$ with a massive H-rich envelopes (e.g., SN1987A and SN1999em).

A strong explosion and a high efficiency for the conversion of the jet energy requires low jet velocities or a low, initial collimation of the jet. With increasing extension of the envelope, the conversion factor increases. Typically, we would expect higher kinetic energies in SNe II compared to SNe Ib/c if a significant amount of explosion energy is carried away by jets. Within the framework of jet-induced SN, the lack of this evidence suggests low jetvelocities. The He, C, O and Si rich layers of the progenitor show characteristic, butterfly-shape structures, and jets bring heavy elements (e.g., ${ }^{56} \mathrm{Ni}$ into the outer layers). Due to the high entropies of the jet material close to the center, this may be a possible site for r-process elements. Moreover, aspherical explosion models show a significantly increased fall-back of material on the central object, e.g., a neutron star, on time scales of minutes to hours which may trigger the delayed formation of a black hole. Fallback and the low velocity material may alter the escape probability for $\gamma$-rays produced by radioactive decay of ${ }^{56} \mathrm{Ni}$ which is critical for mass estimates of ${ }^{56} \mathrm{Ni}$ which are based on late time observations (e.g., SN1998bw). For more details, see $[11,17]$.

\subsection{SN1987A and SN1999em}

In our models for these $\mathrm{SNe}$ II, the jet material stalls within the expanding envelope corresponding to a velocity of $\sim 4500 \mathrm{~km} \mathrm{~s}^{-1}$ during the phase of homologous expansion. In SN1987A, a bump in spectral lines of various elements has been interpreted by material excited by a clump of radioactive ${ }^{56} \mathrm{Ni}$ (Lucy 1988). Within our framework, this bump may be a measure of 

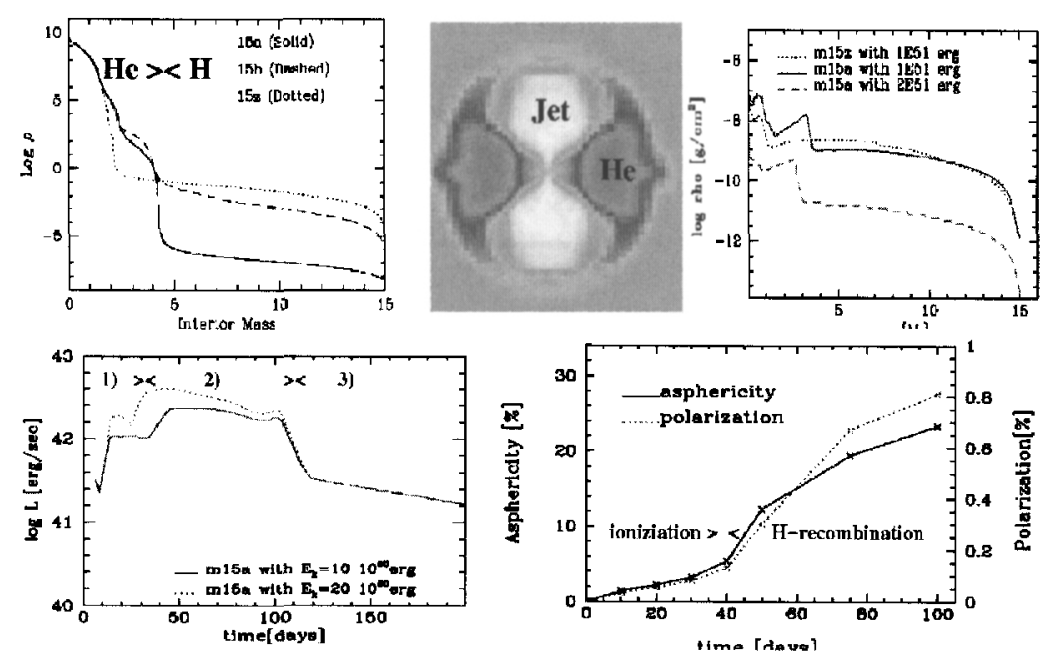

Fig. 3. Polarization produced by an aspherical, chemical distribution for an extreme SN IIP model such as SN1999em (see text).

the region where the jet stalled. This could also explain the early appearance of X-rays in SN1987A which requires strong mixing of radioactive material into the hydrogen-rich layers, and the overall distribution of elements in the resolved HST images of the inner debris of SN 1987A. For both SN1987A and SN1999em, aspherical excitation by hard radiation is found to be crucial to explain the size and presence of the polarization observed early on (Fig. 4). For the extreme SN IIP 1999em (Fig. 3), our model is based on a star with 15 solar masses and an explosion energy of $2 \times 10^{51} \mathrm{erg}$. The initial density profile is given for a star at the final stage of stellar evolution for metallicities $\mathrm{Z}$ of $0.02,0.001$ and 0 (models 15a, 15b, 15z, upper left panel). For the explosion, we use model 15a. In the upper, middle panel, the chemical distribution of He is given at $250 \mathrm{sec}$ for the He-rich layers after the jet material has stalled. The several curves correspond to He mass fractions of $0.0,0.18,0.36$, 0.72 , and 1.0, respectively. The subsequent explosion has been followed in 1-D up to the phase of homologous expansion. In the upper, right panel, the density distribution is given at about 5 days after the explosion. The steep gradients in the density in the upper right and left panels are located at the interface between the He-core and the H-mantel. In the lower, left panel, the resulting bolometric LCs are given for explosion energies of $2 \times 10^{51} \mathrm{erg}$ (dotted line) and $1 \times 10^{51} \mathrm{erg}$, respectively. Based on full 3-D calculations for the radiation and $\gamma$-ray transport, we have calculated the location of the recombination front (in NLTE) as a function of time. The resulting shape of the photosphere is always prolate. The corresponding axis ratio and the polarization seen from the equator are shown (lower, right panel). Note the 

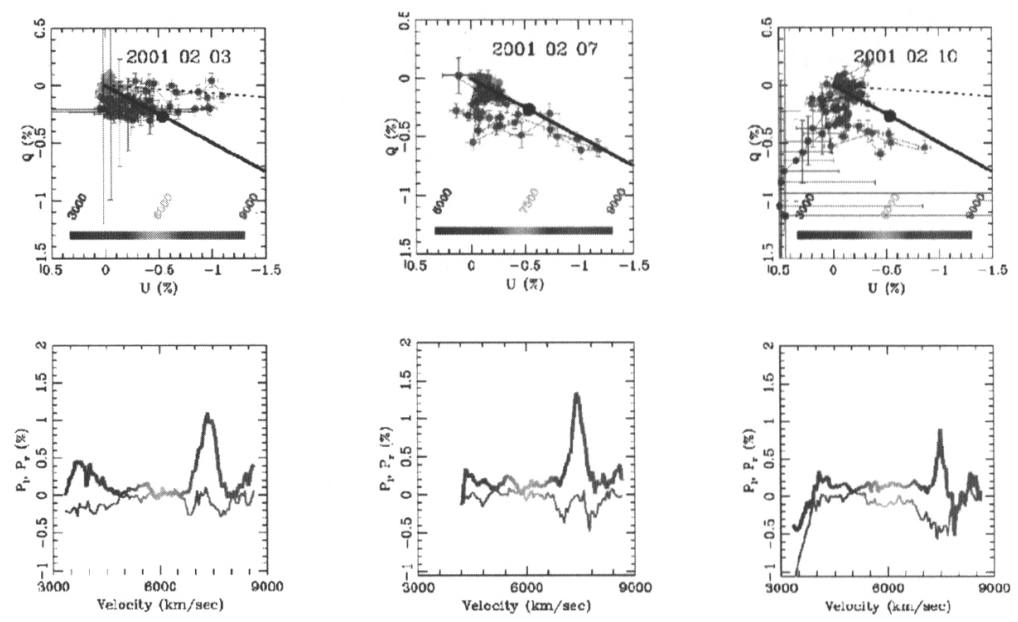

Fig. 4. Spectropolarimetry of SN2002ap at about $-6 \mathrm{~d},-2 \mathrm{~d}$ and +1 relative to maximum light in $\mathrm{V}$. An intrinsic polarization component (shown as a solid dot) is subtracted from the observed Stokes Parameters so that the data points present the intrinsic polarization of the SN. The dashed line illustrates the dominant axis of the polarization. There has been a distinct shift and, perhaps, a small rotation of the axis in the Q-U plane during the early epochs, and the spectra are dominated by FeII, NaI $\mathrm{D}$ and OI. In the spectrum at about $1 \mathrm{~d}$ after maximum, the original, dominant axis has shifted further in the Q-U plane, and a new axis starts to appear at an angle of about $110^{\circ}$ defined by Ca II $\mathrm{H} \& \mathrm{~K}$ and $\mathrm{Ca}$ II IR triplet.

strong increase of the asphericity after the onset of the recombination phase between day 30 to 40 [11]. For the polarization in a massive, H-rich envelope, $P$ is linked to the recombination process and asymmetric excitation.

\section{$2.3 \mathrm{SN}$ 2002ap}

SN 2002ap has attracted much attention because early spectra showed a lack of hydrogen and helium characteristic of SN Ic and broad velocity components $[6,15,21]$, which have been taken as one characteristic of "hypernovae." The nature, existence of, and importance of hypernovae remains to be clarified, and the study of SN 2002ap presents an important opportunity to shed light on the general category of hypernovae. and their relation to typical SNe Ic such as SN1994I. High-quality spectropolarimetry (range 417-860 nm; spectral resolution $1.27 \mathrm{~nm}$ ) of SN 2002ap was obtained with the ESO Very Large Telescope Melipal (+ FORS1) at 3 epochs that correspond to $-6,-2$, and +1 days for a $\mathrm{V}$ maximum of 9 Feb 2002. The polarization spectra show three distinct broad $(\sim 100 \mathrm{~nm})$ features at $\sim 400,550$, and $750 \mathrm{~nm}$ that evolve in shape, amplitude and orientation in the Q-U plane. The continuum polarization grows from nearly zero to $\sim 0.2$ percent. The $750 \mathrm{~nm}$ feature is 
polarized at a level $\geq 1$ percent. We identify the 550 and $750 \mathrm{~nm}$ features as $\mathrm{Na} \mathrm{I} \mathrm{D} \mathrm{and} \mathrm{OI} \lambda 777.4$ moving at about $20,000 \mathrm{~km} \mathrm{~s}^{-1}$. The blue feature may be Fe II. We interpret the polarization evolution in terms of the impact of a bipolar flow from the core that is stopped within the outer envelope of a carbon/oxygen core and, consequently, the $\mathrm{Ca}$ features show up only at about maximum light. The interpretation of a stalled jet is also supported by IR-spectra taken by C. Gerardy and M. Meikle (2002, private communication) which show strong CI lines (940.5 and 1070. nm) at expansion velocities of $\sim 15,000$ to $25,000 \mathrm{~km} \mathrm{~s}^{-1}$ but not, as in SNe Ia, the strong 1600 to 1900 $\mathrm{nm}$ feature due to $\mathrm{Fe} / \mathrm{Co} / \mathrm{Ni}$. Although the symmetry axis remains fixed, the photosphere retreats by different amounts in different directions due to the asymmetric velocity flow and density distribution geometrical blocking effects leading to a continuous shift with time of the main axis of polarization. At about maximum light, the appearance of an additional axis in a Q-U plane due to Ca and processed material indicates a second axis of symmetry. Qualitatively and within the picture of jet-induced supernovae this may be explained by bipolar-jets which are not perfectly aligned and, thus, produce a kick of the central region (e.g., neutron star and processed material). Detailed radiation-hydro calculations are under way. We conclude that the features that characterize SN 2002ap, specifically its high velocity, can be accounted for in an asymmetric model with a larger ejecta mass than SN 1994 I such that the photosphere remains longer in higher velocity material. The characteristics of hypernovae may be the result of orientation effects in a mildly inhomogeneous set of progenitors, rather than requiring an excessive total energy or luminosity. In the analysis of asymmetric events with spherically symmetric models, it is probably advisable to refer to "isotropic equivalent" energy, luminosity, ejected mass, and nickel mass. This aspect may also be relevant for the interpretation of the hypernova SN1998bw.

Acknowledgement. The authors are grateful to the European Southern Observatory for the generous allocation of observing time. They especially thank the staff of the Paranal Observatory for their competent and un-tiring support. This work was supported in part by NASA Grant NAG5-7937.

\section{References}

1. G.S. Bisnovatyi-Kogan: Sov. Astron. 14, 652 (1971)

2. R.L. Bowers R.L., J.R. Wilson: J.R. Astrophys. J. Suppl. 50, 115 (1982)

3. P. Colella, P.R. Woodward: J. Comput. Phys. 54, 174 (1984)

4. M. Cropper et al. : Mon. Not. R. Astron. Soc. 231, 685 (1988)

5. R.A. Fesen, K.S. Gunderson: Astrophys. J. 470, 967 (1996)

6. A. Gal-Yam, O. Shemmer, J. Dann: IAUC 7811 (2002)

7. P. Höflich: Pub. Astron. Soc. Pacific 7, 434 (1988)

8. P. Höflich: Astron. Astrophys. 246, 481 (1991) 
9. P. Höflich: Astrophys. J. 443, 89 (1995)

10. P. Höflich, J.C. Wheeler, D. Hines, S. Trammell: Astrophys. J. 459, 307 (1995)

11. P. Höflich, A. Khokhlov, L. Wang: In: $20^{\text {th }}$ Texas Conf. on Rel. Astro. (AIP Conf. Proc. 586, 2001), p. 459

12. P. Höflich: astro-ph 0207103 (2002)

13. J.P. Hughes, C.E. Rakowski, D.N. Burrows, P.O. Slane: Astrophys. J. Lett. 528, L109 (2000)

14. D.J. Jeffrey: Astrophys. J. 375, 264 (1991)

15. K. Kinugasa et al. : IAUC 7811 (2002)

16. A.M. Khokhlov: J. Comput. Phys. 143, 519 (1998)

17. A. Khokhlov, P. Höflich, E. Oran, C. Wheeler, L. Wang: Astrophys. J. Lett. 524, L107 (1999)

18. J.M. LeBlanc, J.R. Wilson: Astrophys. J. 161, 541 (1970)

19. D.C. Leonard, A.V. Filippenko, A.J. Barth, T. Matheson: Astrophys. J. 536, $239(2000)$

20. L.B. Lucy: In: $4^{\text {th }}$ Mason Conference, ed. M. Kafatos (Cambridge Univ. Press: Cambridge 1998) p. 323

21. P. Meikle et al. : IAUC 7811 (2002)

22. R.H. Mendez et al. : Astrophys. J. 334, 295 (1998)

23. R. Mönchmeyer, G. Schäfer, E. Müller, R.E. Kates: Astron. Astrophys. 246, 417 (1991)

24. B.E. Schaefer et al. : Astrophys. J. 588, 387 (2003)

25. K.Z. Stanek et al. : Astrophys. J. Lett. 591, L17 (2003)

26. O. Straniero, A. Chieffi, M. Limongi: Astrophys. J. 490, 425 (1997)

27. R. Strom, H.M. Johnston, F. Verbunt, B. Aschenbach: Nature 373, 587 (1995)

28. E.M.D. Symbalisty: Astrophys. J. 285, 729 (1984)

29. S. Trammell, D. Hines, J.C. Wheeler: Astrophys. J. 414, 21 (1993)

30. L. Wang, J.C. Wheeler, Z. Li, A. Clocchiatti: Astrophys. J. 467, 435 (1996)

31. L. Wang, A. Howell, P. Höflich, J.C. Wheeler: Astrophys. J. 550, 1030 (2001)

32. L. Wang, D. Baade, P. Höflich, J.C. Wheeler: Astrophys. J. 592, 457 (2003)

33. L. Wang et al. : Astrophys. J. 591, 1100 (2003)

34. S.E. Woosley, A.I. MacFadyen: Astron. Astrophys. Suppl. 138, 499 (1999) 